\title{
Efectos del entrenamiento en intervalos de alta intensidad en adultos mayores: una revisión sistemática
}

\section{Effects of high-intensity interval training in older adults: a systematic review}

\author{
Nicole B. Fritz Silva ${ }^{1-2}$ \\ 1 Departamento de Salud, Kinesiología, Universidad de Los Lagos. Chile. \\ 2 Grupo de Investigación en Prevención y Salud en el Ejercicio y el Deporte, Universidad de Valencia. España.
}

\section{CORRESPONDENCIA:}

Nicole Beatriz Fritz Silva

nicole.fritz@ulagos.cl

Recepción: diciembre 2019 • Aceptación: septiembre 2020

\section{CÓMO CITAR EL ARTÍCULO:}

Fritz, N.B. (2021). Efectos del entrenamiento en intervalos de alta intensidad en adultos mayores: una revisión sistemática. Cultura, Ciencia y Deporte, 16(48), 187-198. http://dx.doi.org/10.12800/ ccd.v16i48.1627

\section{Resumen}

El objetivo de este estudio fue realizar una revisión sistemática de la literatura sobre las características de los programas de entrenamiento basados en la modalidad de intervalos de alta intensidad (HIIT, siglas en inglés) en adultos mayores, y a su vez, describir los efectos sobre la capacidad física y funcional en esta población. Se realizó una búsqueda de literatura de 5 bases de datos (DIALNET, DOAJ, Elsevier, PubMed y Web of Science). Los criterios de inclusión incluyeron estudios experimentales aplicados en personas mayores de 60 años. La escala PEDro se utilizó para la evaluación de la calidad de los estudios elegibles. La búsqueda identificó 126 artículos y se evaluaron 12 artículos en texto completo. Todos los estudios eran de buena calidad metodólogica y tenían un bajo riesgo de sesgo. En conclusión, el entrenamiento HIIT ha sido aplicado por un periodo de 6 a 16 semanas, en la modalidad "HIIT-aeróbico" con efectos positivos sobre la capacidad aeróbica y funcional de las personas mayores, además ha reducido la carga patológica en artritis reumatoidea, obesidad y/o dinapenia.

Palabras clave: ejercicio, capacidad funcional, anciano, intensidad, entrenamiento interválico.

\section{Summary}

The objective of this study was to do a systematic review of the literature about the characteristics of training programs based on the high intensity interval modality (HIIT) in elderly, and at the same time, to describe the effects on physical and functional capacity in this population. A literature search of 5 databases (DIALNET, DOAJ, Elsevier, PubMed and Web of Science) was performed. The inclusion criteria included experimental studies applied in people older than 60 years. The PEDro scale was used for quality evaluation of eligible studies. The search identified 126 articles and evaluated 12 articles in full text. All studies were of good methodological quality and had a low risk of bias. In conclusion, HIIT training has been applied for a period of 6 to 16 weeks, in the "HIIT-aerobic" modality with positive effects about the aerobic and functional capacity of the elderly, it has also reduced the pathological burden in rheumatoid arthritis, obesity and / or dynapenia.

Key words: exercise, functional capacity, elderly, intensity, interval training 


\section{Introducción}

El ejercicio físico es considerado la piedra angular para reducir los efectos del sedentarismo, fomentar la salud y el bienestar en los adultos mayores (Landi, Marzetti, Martone, Bernabei \& Onder, 2014), capaz de generar efectos positivos incluso en quienes nunca han realizado actividad física (Berk, Hubert \& Fries, 2006 \& Hamer, Lavoie \& Bacon, 2014). Es por lo que el Colegio Americano de Medicina Deportiva recomienda en personas mayores de 65 años, y más, acumular un total de 150 minutos (5 veces a la semana) de ejercicio aeróbico a moderada intensidad o 60 minutos (3 veces a la semana), si esta actividad es realizada a intensidad vigorosa (Chodzko-Zajko et al., 2009). Dentro de los beneficios que se pueden nombrar por la práctica de ejercicio regular se encuentran la disminución del riesgo de desarrollo de enfermedades cardiometabólicas, obesidad, caídas, deficiencias cognitivas, osteoporosis, debilidad muscular e incluso combatir la mortalidad prematura (McPhee et al., 2016; Kim et al., 2012 \& Manini et al., 2006). Sin embargo, para que los individuos se adhieran a largo plazo a este tipo de intervenciones es fundamental la prescripción personalizada, teniendo en consideración además de la edad, la capacidad física/ funcional inicial (Hautier \& Bonnefoy, 2007). Buscando precisamente dicha adherencia y considerando que la población de adultos mayores son el grupo de edad más sedentario, con un promedio de conducta sedentaria de un $65-80 \%$ de su tiempo de vigilia (Wullems, Verschueren, Degens, Morse \& Onambélé, 2006), es que actualmente los autores han estudiado la implementación de los programas de entrenamiento basados en la aplicación de intervalos de alta intensidad (High Intensity Interval Training, HIIT en inglés) como una alternativa al entrenamiento tradicional de fuerza muscular y capacidad aeróbica en esta población.

El entrenamiento HIIT es una modalidad que se basa en la realización de series de ejercicios de alta intensidad, con intervalos pasivos o de baja intensidad (Buchheit \& Laursen, 2013) que, en comparación al ejercicio de moderada intensidad tradicional, acumula un mayor volumen de trabajo a mayor intensidad en una sola sesión. Es precisamente esta característica lo que lo ha convertido en una alternativa atractiva para las poblaciones sedentarias que pueden ser más propensas a incorporar episodios breves de actividad física vigorosa en un día (Gibala, Little, MacDonald \& Hawley, 2012). Se diferencia del entrenamiento de sprint repetidos (SIT, siglas en inglés) principalmente porque SIT implica series repetidas de ejercicio de alta intensidad, igual o superior a la velocidad máxima intercalados con períodos de recuperación de menor duración que HIIT (Billat, 2001). Existen dos tipos de implementación del HIIT, uno hace referencia al "HIIT contrarresistencia", en que el entrenamiento de intervalos utiliza movimientos corporales, pesos libres o dispositivos para actividades de alta resistencia (Kilpatrick, Jung, \& Little, 2014 \& McRae et al., 2012) y el otro, es el "HIIT aeróbico" en que se utilizan modalidades tradicionales de ejercicio aeróbico como correr y andar en bicicleta, siendo esta última la más frecuentemente utilizada, debido a que permite una evaluación más precisa del trabajo implementado (Helgerud et al., 2007 \& Kilpatrick, Jung, \& Little, 2014). Independiente de la modalidad seleccionada, dentro de los beneficios que se han documentado en la literatura para la población adulta en general, en condiciones de salud y enfermedad se encuentran mejoras a nivel de la capacidad aeróbica, función física, composición corporal, salud metabólica y cardiovascular (Adamson, Lorimer, Cobley, Lloyd \& Babraj, 2014; Campbell et al., 2019; Carrasco-Poyatos \& Reche-Orene, 2018; Gibala \& McGee, 2008; Gibala et al., 2012; Maillard, Pereira \& Boisseau, 2018; Wisløff et al., 2007 \& Wisløff et al., 2009). Además, el Colegio Americano de Medicina Física y Deporte recomienda incorporar 1 a 2 sesiones HIIT en el programa de entrenamiento semanal habitual de una persona mayor con una o más patologías crónicas a fin de estimular sus adaptaciones fisiológicas (Brad, 2013 \& Campbell et al., 2019).

Por todo lo anteriormente descrito, el objetivo de la presente revisión bibliográfica es conocer las características de los programas de entrenamiento basados en la modalidad HIIT que han sido estudiados en la literatura científica en población adulto mayor y, a su vez, describir los efectos sobre la capacidad física y funcionalidad en esta población.

\section{Metodología}

Fuentes y búsqueda en la literatura

La revisión se realizó durante los meses de septiembre a noviembre de 2019, aplicando las pautas establecidas de la declaración PRISMA (Moher, Liberati, Tetzlaff, Altman \& Prisma Group, 2010). Esta revisión no cuenta con un número de protocolo de registro. Las bases de datos utilizadas fueron DIALNET, DOAJ, Elsevier, PubMed y Web of Science (WOS) seleccionando artículos con una antigüedad de 10 años desde octubre 2009 hasta octubre del año 2019. Se emplearon los siguientes términos $\mathrm{MeSH}$ en inglés combinados con diferentes operadores booleanos en la búsqueda: (high intensity interval training OR high intensity 


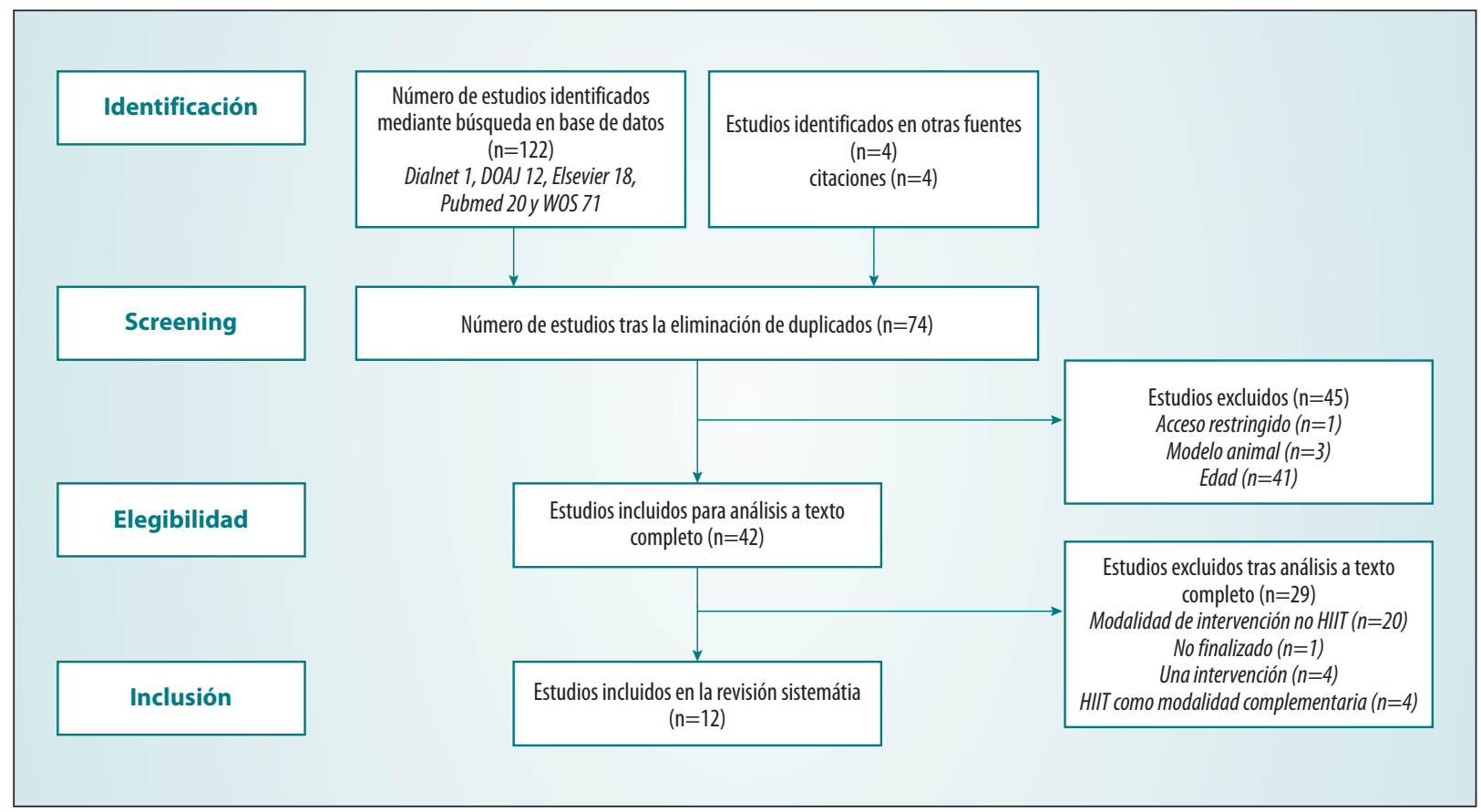

Figura 1. Diagrama de flujo PRISMA.

Tabla 1. Criterios de elegibilidad para incluir los estudios en la revisión.

\begin{tabular}{ll}
\hline Tópico & Criterios de inclusión \\
\hline Participantes & Adultos mayores de 60 años y más, sanos y con patología diagnosticada. \\
Intervenciones & HIIT, prescripción del HIITT detallada e intervención mayor a 1 sesión. \\
Comparación & No intervención o control, entrenamiento diferente al HIIT, utilización de suplementación alimentaria. \\
Outcome o resultados & Resultados primarios: capacidad física, funcionalidad y/o modificación condición de salud inicial. \\
Diseño & Ensayos clínicos aleatorizados, estudios experimentales o de cohorte. \\
\hline & Criterios de exclusión \\
\hline Participantes & Estudios en animales y personas menores de 60 años. \\
Intervenciones & Entrenamiento de intervalos de alta intensidad sprint. \\
Comparación & Que analizaran los efectos del HIIT complementario a la aplicación de otra modalidad de entrenamiento. \\
Diseño & Conferencias, disertaciones, tesis y publicaciones no arbitradas por pares. \\
\hline
\end{tabular}

intermittent exercise) AND (older adults OR elderly). La búsqueda se limitó a estudios originales de revistas disponibles en texto completo, en el idioma inglés y español de acceso abierto, además, fueron contactados mediante correo electrónico los autores para solicitar aquellos artículos que no estaban disponibles para descarga gratuita en la web. Los artículos obtenidos tras esta búsqueda inicial se seleccionaron de acuerdo con los siguientes criterios.

\section{Criterios de inclusión y exclusión}

La búsqueda de títulos relevantes para el objetivo de este estudio fue realizada por un revisor (N.F.). Si hubo incertidumbre con respecto a la elegibilidad de un estudio, se obtuvo el resumen y se agregó a la siguiente fase de la revisión para mayor aclaración. Luego, los resúmenes se seleccionaron para determinar su elegibilidad considerando 4 puntos claves: (1) diseño experimental, (2) participantes adultos mayores, (3) intervención HIIT y (4) resultados sobre la capacidad física, funcional y/o variación de la condición de salud inicial. Los resúmenes elegibles se incluyeron en la etapa de recuperación de texto completo solo si cumplían los 4 criterios descritos anteriormente. En el caso de necesitar aclaración sobre alguno de estos criterios, el revisor consultó con un colaborador externo (P.V.). Posteriormente, se seleccionaron los manuscritos de texto completo, aplicando los criterios de inclusión y exclusión (Tabla 1). Esto produjo un número final de estudios elegibles para la fase de extracción de datos y en caso de duda o desacuerdo, se consultó a 2 revisores externos (P.V. y M.F.). 


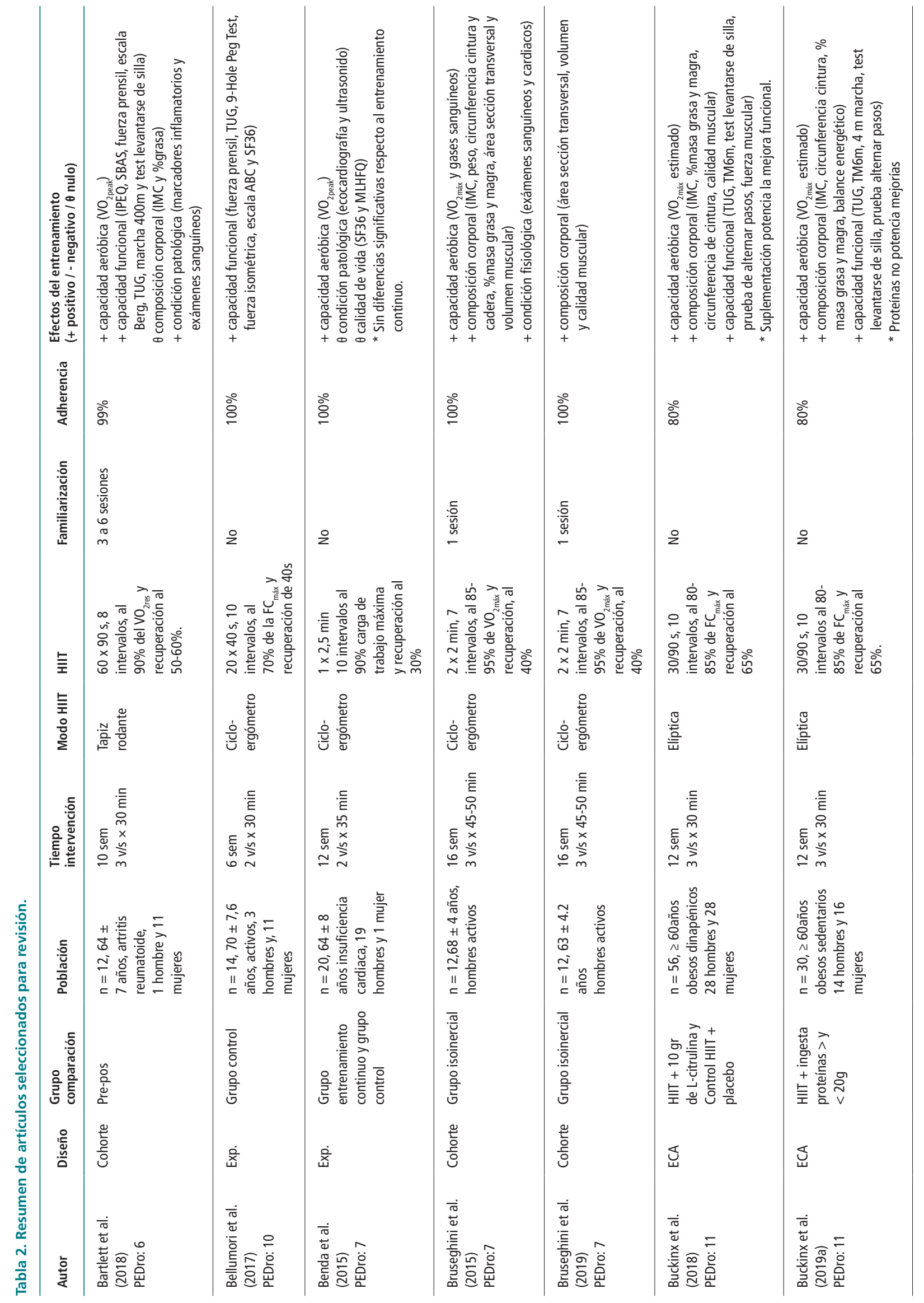




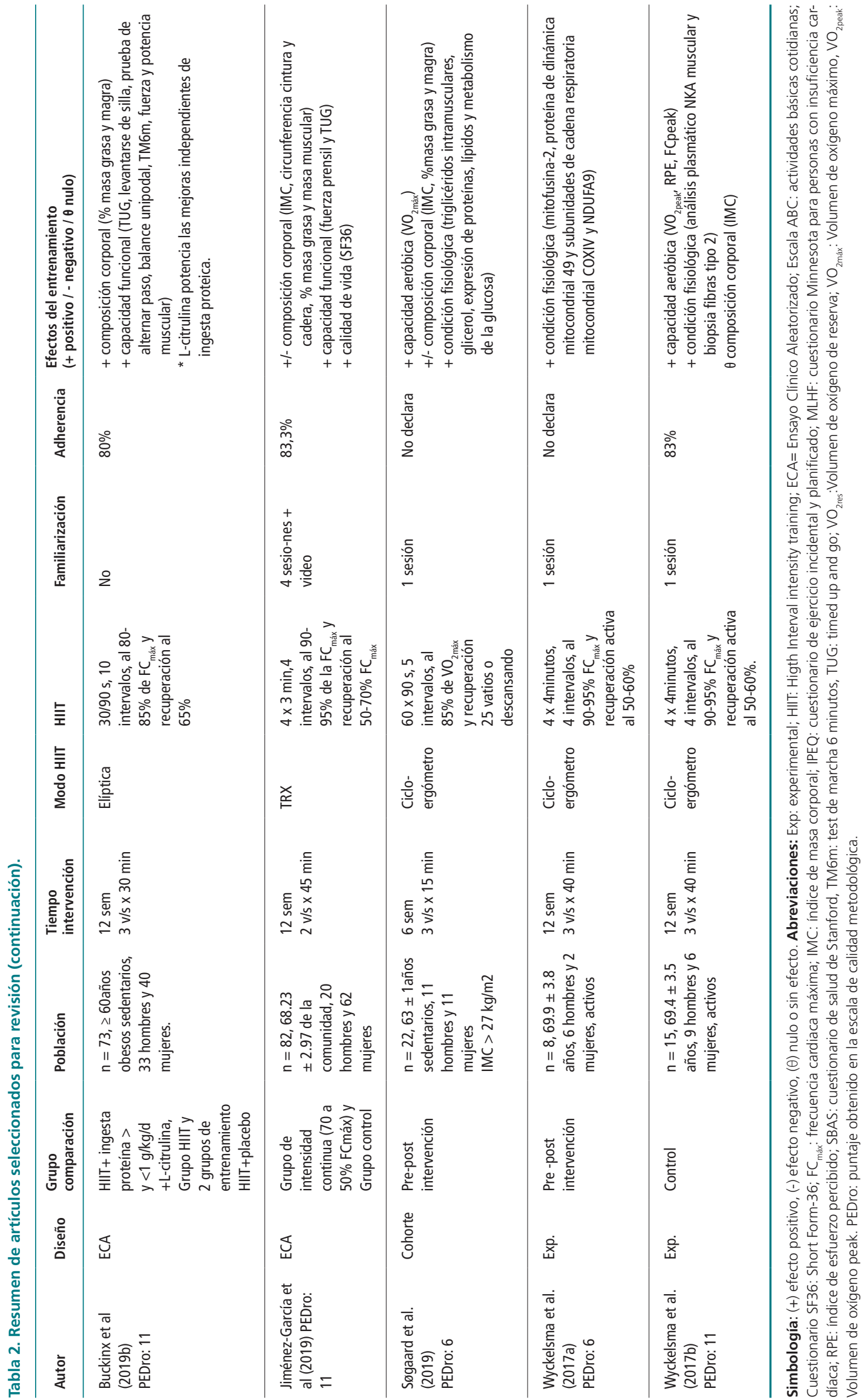




\section{Extracción de datos}

La efectividad de la intervención HIIT se determinó a través de los resultados informados por los estudios para capacidad física, funcional y/o variación de la condición de salud inicial. La extracción de los datos fue realizada por el revisor principal (N.F.) y dos revisores externos (P.V. y M.F.) quienes velaron por la correcta extracción del contenido y nivel de acuerdo.

\section{Evaluación de calidad metodológica}

La calidad metodológica de los estudios se analizó utilizando la "escala PEDro", validada por Maher, Sherrington, Herbert, Moseley y Elkins (2003). Los estudios con una puntuación mayor o igual 6 puntos fueron consideraron de alta calidad metodológica, mientras que una puntuación menor fue considerada de baja calidad.

\section{Resultados}

Las características de los artículos seleccionados, características de la intervención, adherencia y efectos del entrenamiento se resumen en la Tabla 2.

\section{Selección de artículos}

Se seleccionaron 12 artículos, el número de estudios identificados y excluidos puede observarse en la Figura 1. Inicialmente fueron identificados 122 artículos de las diferentes bases de datos consultadas y 4 se incorporaron externamente tras revisar la bibliografía de dichos artículos. Tras la eliminación de estudios duplicados se inició la revisión de 74 artículos aplicando los criterios de selección.

Todos los estudios fueron escritos en inglés y publicados en un rango menor a 5 años (2015 a 2019). La mayoría fueron de tipo experimental, en lo particular ensayos clínicos aleatorizados y compararon el entrenamiento HIIT con un grupo control pasivo, que no realizó entrenamiento $(n=3)$ o activo, que permitió comparaciones con otras modalidades de intervención de ejercicio $(n=4)$ y/o modificación en la ingesta nutricional $(n=3)$. Los autores que publicaron más de un artículo fueron Buckinx (3 publicaciones), Bruseghini y Wyckelsma con 2 publicaciones cada uno.

Los resultados de la evaluación de calidad de cada estudio a través de la escala PEDro se presentan en la Tabla 1. Los 12 estudios seleccionados fueron de buena calidad metodológica y tuvieron un bajo riesgo de sesgo, con puntajes que van de 6 a 11 puntos en la escala PEDro.
Población objetivo

De los artículos seleccionados sólo 4 consideraron una muestra mayor o igual a 30 participantes seleccionando adultos mayores compensados medicamente. En cuanto a las características de la población de estudio, 3 autores en 5 investigaciones diferentes estudiaron el efecto del entrenamiento HIIT en condiciones patológicas como son la artritis reumatoide (Bartlett et al., 2018), insuficiencia cardiaca (Benda et al., 2015) y obesidad y/o dinapenia (Buckinx et al., con 3 estudios), mientras que en 5 trabajos se intervinieron adultos mayores activos o moderadamente activos. Respecto a la cantidad de participantes que completaron la intervención 9 artículos mencionan una adherencia alta ( $\geq 80 \%)$, solo 2 no reportan la adherencia como variable a controlar o registrar. El único autor que declara complicaciones médicas producto del entrenamiento HIIT es Wyckelsma et al. (2017b) pero fueron episodios vasovagales leves sin más incidentes posteriores.

\section{Tiempo de intervención}

Se realizaron con una duración de intervención que osciló entre un mínimo de 6 y máximo de 16 semanas con una frecuencia de 2 a 3 veces a la semana, siendo la mayoría (9 estudios) 3 veces a la semana en días no consecutivos. Además, en su mayoría los autores realizaron mínimo una sesión de familiarización previa a las semanas de entrenamiento, destacando el estudio de Bartlett et al. (2018) quienes realizaron de 3 a 6 sesiones y el de Jiménez-García et al. (2019) con 4 sesiones, los demás estudios no indica la realización de familiarización. En cuanto al tiempo de intervención todos los estudios a excepción del de Søgaard et al. (2019) tuvieron una duración de la sesión de 30 a 45 minutos.

\section{Características del protocolo HIIT}

En cuanto a la modalidad preferida para realizar el entrenamiento fue "HIIT aeróbico" $(n=11)$, de los cuales 7 estudios de 5 autores diferentes utilizaron cicloergómetro; los demás trabajos utilizaron elíptica (3 estudios de Buckinx et al.) y tapiz rodante $(\mathrm{n}=2)$, mientras que el estudio restante de Jiménez-García et al. (2019) utilizó la modalidad "HIIT contrarresistencia” a través de TRX (Tabla 2). Respecto a la prescripción de los intervalos de alta intensidad, todos los artículos a excepción del de Bellumori et al. (2017), entrenaron a intensidades sobre el 80\% ( $n=11)$ con un rango de 80 a 95\%. Respecto a la relación tiempo de 
alta intensidad y reposo activo las relaciones oscilaron entre $1: 1(n=4) ; 1: 1.5(n=3), 1: 2(n=1), 1: 3(n=$ 3) y en sólo 1 trabajo el tiempo de alta intensidad fue superior al tiempo de reposo activo con una relación de 2:1.5 (Jiménez-García et al. 2019). Los tiempos de dichos intervalos se distribuyeron desde un mínimo de $30 \mathrm{~s}$ hasta los 4 minutos, con un número intervalos entre 4 a 10 .

\section{Efectos del entrenamiento HIIT}

Los efectos del entrenamiento HIIT para cada uno de los artículos revisados se resumen en la Tabla 2. Con el objeto de resumir los hallazgos, se han decidido mostrar los efectos del entrenamiento HIIT en adultos mayores en 5 categorías: capacidad aeróbica con 7 artículos, composición corporal con 8 artículos, capacidad funcional con 6 artículos, calidad de vida con 3 artículos y sobre condiciones patológicas o fisiológicas con 5 artículos. En cuanto a la categoría capacidad aeróbica, el $100 \%$ de los artículos reportaron mejoras significativas independiente del tiempo de intervención, condición funcional de la población intervenida, características del HIIT y control o modificación alimentaria. De estos estudios, 5 artículos (Bartlett et al. 2018, Benda et al., 2015, Bruseghini et al., 2015, Søgaard et al., 2019 y Wyckelsma et al., 2017b) no modificaron la ingesta alimentaria de los participantes; mientras que 2 estudios de Buckinx et al., en adultos mayores si lo hicieron a través del control de ingesta proteica (Buckinx et al., 2019a) y suplementación con citrulina (Buckinx et al., 2018).

Respecto a los efectos del entrenamiento HIIT sobre parámetros de composición corporal la evidencia es controvertida, debido a que de un total de 8 artículos que incorporaron diversas variables para evaluar cambios en este componente, 4 artículos reportan efectos positivos (Bruseghini et al 2015; Bruseghini, Capelli, Calabria, Rossi \& Tam, 2019; Buckinx et al., 2019 y Buckinx et al., 2018), de estos se debe considerar que los dos estudios de Buckinx et. al, confirman estos efectos con independencia de la ingesta de proteínas o suplementación con citrulina en adultos mayores que previamente tenían alteración en la composición corporal. Por el contrario, 2 estudios (Søgaard et al., 2019 \& Jiménez-García et al., 2019) no reportan efectos, mientras que 2 artículos de Barlett et al. (2018) y Wyckelsma et al. (2017b) no presentaron resultados concluyentes.

La totalidad de estudios que caracterizaron la capacidad funcional con diferentes variables extraídas de la aplicación de diversas pruebas validadas y de utilización frecuente en personas mayores, reportan mejoras en la población de estudio. Dichas mejoras significativas han sido en parámetros de fuerza muscular de extremidad superior $(n=5)$ e inferior $(n=4)$ $y$ en variables que caracterizan la movilidad funcional a través de la aplicación de una o más pruebas de marcha, balance y/o coordinación ( $\mathrm{n}=6$ ) (ver Tabla 2$) .3$ artículos reportan cambios significativos en todas las dimensiones antes nombradas de la capacidad funcional (Bellumori et al.,2007, Buckinx et al., 2018 y Buckinx et al., 2019), 3 artículos en dos dimensiones, que en el caso del estudio de Buckinx et al., (2019b) y Jiménez-García et al., (2019) fueron en fuerza muscular de extremidades superiores y movilidad funcional, mientras que para el estudio de Bartlett et al. (2018) también fueron en movilidad funcional pero en fuerza de extremidades inferiores.

Solamente 3 autores evaluaron los cambios sobre la calidad de vida tras el programa de intervención HIIT con resultados diferentes: Bellumori et al., (2017) y Benda et al., (2015), no lograron mejoras significativas en adultos mayores con patologías, mientras que el estudio de Jiménez-García et al. (2019) que intervino a adultos mayores de la comunidad, es el único de los estudios que reporta beneficios significativos y con un alto tamaño del efecto después del entrenamiento HIIT en comparación con los grupos que entrenaron a moderada intensidad y el grupo control.

Por último, en cuanto a la categoría de condiciones patológicas o fisiológicas, el entrenamiento HIIT demostró disminuir la carga mórbida de adultos mayores con diagnóstico de artritis reumatoidea (Bartlett et al, 2018) y obesidad dinapénica (Buckinx et al., 2018), pero sin efectos significativos sobre la condición patológica de aquellos que tenían diagnóstico de insuficiencia cardiaca (Benda et al., 2015). Sin embargo, existieron estudios que reportaron mejoras significativas en condiciones que podrían volverse potencialmente patológicas como son la sensibilidad a la insulina (Søgaard et al., 2019), factores de riesgo que predisponen al síndrome metabólico (Bruseghini et al., 2015) y parámetros de disfunción mitocondrial (Wyckelsma et al., 2017a).

\section{Discusión}

La presente revisión demuestra que el entrenamiento HIIT es implementado por un tiempo de 6 a 16 semanas, con una frecuencia de 2 a 3 veces semanales con una duración de 30 a 45 minutos por sesión, en cuanto a las características del HIIT, la modalidad más utilizada es el "HIIT aeróbico" con aplicación de intensidades submáximas de 80 a 95\%. En cuanto a 
sus efectos, se reporta en la literatura mejoras en la capacidad aeróbica y funcional de las personas mayores, sin consenso en los artículos incluidos en esta revisión en la mejora de parámetros de composición corporal y calidad de vida, sobre todo en condiciones patológicas. Además, ha demostrado ser beneficioso en reducir la carga patológica en personas mayores con artritis reumatoidea, obesidad y/o dinapenia, a su vez potencialmente efectivo en combatir la sensibilidad a la insulina, el síndrome metabólico y disfunción mitocondrial en adultos mayores sanos.

\section{Efectos del entrenamiento HIIT sobre la capacidad aeróbica}

Los artículos analizados informan mejorías cercanas al $10 \%$ en variables que caracterizaron la capacidad aeróbica en un tiempo de 10,12 y 16 semanas con una frecuencia semanal de 3 veces a la semana en 6 de 7 artículos. Dentro de los estudios que no hicieron modificaciones en la ingesta alimentaria $(n=4)$ se encuentran el estudio de Bartlett et al., (2018) con aumentos en el $\mathrm{VO}_{2 \text { máx }}$ absoluto y relativo en el orden de un $9 \%$ tras 10 semanas de intervención, mientras que el estudio de Bruseghini et al., (2015) refiere mejoras de alrededor de un $7 \%$ en el $\mathrm{VO}_{2 \max }$ y mejoras significativas en el umbral ventilatorio evaluado con el punto de compensación respiratorio de un $19 \%$, tras 4 meses de intervención; mismo porcentaje de mejoría en el $\mathrm{VO}_{2 \text { peak }}(7 \%)$ reporta el estudio de Benda et al., (2015) pero en adultos mayores con insuficiencia cardiaca en un tiempo menor de intervención (12 semanas); mientras que el estudio de Wyckelsma et al., (2017b) en el mismo tiempo de intervención reporta mayores mejoras $\left(+16 \% \mathrm{VO}_{2 \text { peak }} ; \mathrm{p}=0,002\right)$ en adultos mayores activos con un volumen semanal de entrenamiento mayor (12 semanas, $3 \mathrm{v} / \mathrm{s}$ ). Por lo tanto, existe una tendencia a mayores mejoras en la capacidad aeróbica relacionadas con una duración del programa mayor o igual a 10 semanas de intervención. En el caso de los estudios que controlaron o modificaron la ingesta alimentaria en adultos mayores con alteración de la composición corporal (obesidad y/o dinapenia) se encuentran dos trabajos de Buckinx et al., (2018 y 2019a) de 12 semanas de intervención. Tras revisar estos estudios es posible ver mejoras de un $15,6 \%$ en el $\mathrm{VO}_{2 \max }$ estimado en los grupos que tuvieron una ingesta proteica diaria < 20 gramos versus $10,1 \%$ de mejora en las personas mayores que superaron los 20 gramos (Buckinx et al., 2019a), mientras que en el estudio en que las personas mayores fueron suplementadas con citrulina las mejoras en el $\mathrm{VO}_{2 \max }$ estimado fueron de un $11,8 \%$ versus $9,1 \%$ en las personas que consumie- ron el placebo (Buckinx et al., 2018). Por último, el único estudio que reportó mejoras menores a las antes mencionadas fue el de Søgaard et al. (2019) ( $\mathrm{VO}_{2 \text { máx }}$ : $+5,6 \%$ ), sin embargo, los autores intervinieron con un volumen de entrenamiento semanal menor a todos los estudios antes mencionados.

\section{Efectos del entrenamiento HIIT sobre la composición corporal}

Respecto a los efectos del entrenamiento HIIT sobre parámetros de composición corporal la evidencia es controvertida. De un total de 8 artículos que incorporan esta variable, 2 no reportan efectos significativos sobre los parámetros de composición corporal como es el peso (Bartlett et al., 2018) e índice de masa corporal y porcentaje de masa grasa (Wyckelsma et al., 2017b). 2 estudios presentaron datos disidentes, donde Søgaard et al. (2019) coincide en el nulo efecto sobre el índice de masa corporal, pero demuestra un efecto significativo para la reducción de masa grasa y aumento de la masa libre de grasa, mientras que Jiménez-García et al., (2019) señala lo contrario, demostrando una disminución significativa del índice de masa corporal, sin diferencias significativas en variables que caracterizaron masa grasa y masa muscular.

Por último, los estudios de Bruseghini et al., demuestran una reducción significativa en el porcentaje de grasa total y visceral (Bruseghini et al., 2015) y aumento en el área de sección transversal y volumen muscular (Bruseghini et al., 2015 y 2019) y los estudios de Buckinx et al., confirman estos hallazgos tras reportar una reducción de los perímetros de cintura y cadera independiente de la ingesta de proteínas (Buckinx et al., 2019) y suplementación con citrulina (Buckinx et al., 2018), sin embargo, sugieren que en adultos mayores con obesidad en que su ingesta proteica no sobrepase 1 gramo por kilógramo diario de proteínas, lograrían una mayor mejora en composición corporal y fuerza muscular si se suplementan con citrulina (Buckinx et al., 2019a).

Efectos del entrenamiento HIIT sobre la capacidad funcional

Respecto a los efectos reportados para la fuerza muscular de tren superior los 5 artículos que estudiaron esta variable difieren en el porcentaje de mejora documentada. Dentro de los factores que podrían influenciar este resultado se encuentran, además de la diversidad física basal, debido a que en el caso de los estudios de Buckinx et al., los adultos mayores tenían obesidad y/o dinapenia, se encuentra la acción que 
cumplen los músculos de extremidad superior para la ejecución del entrenamiento en el equipamiento seleccionado por los autores: Bellumori et al., cicloergómetro, Jiménez-García et al. (2019), suspensión en TRX y Buckinx et al., elíptica.

El estudio de Bellumori et al. (2017) indica mejoras de un $47 \%$ en la fuerza isométrica de los extensores del codo tras 6 semanas de intervención, superior a los demás autores, esto se puede deber a la función que ejecuta la musculatura para la mantención de la posición sobre el cicloergómetro. El estudio de Jiménez- García et al. (2019) reporta un aumento significativo de alrededor de un $12 \%$ ( $\mathrm{p}=0.002$ ) de la fuerza isométrica prensil en adultos mayores de la comunidad, mientras que los estudios de Buckinx et al., logran una mejoría de la fuerza isométrica de extremidad superior de un $5 \%$ en personas con obesidad y/o dinapenia independiente de la ingesta de proteínas diaria (Buckinx et al., 2019a; Bucknick et al., 2019b) y la suplementación con citrulina (Buckinx et al., 2019b; Bucknick et al.,2018). Sin embargo, en los grupos con suplementación con citrulina, existe un mayor porcentaje de personas que responden al entrenamiento HIIT (80\% grupo suplementado con Citrulina versus $50 \%$ placebo) y dicha suplementación potenció las mejoras alcanzadas por el HIIT de manera clínicamente significativa (suplementación con citrulina 9,3\% versus placebo PLA: 3,3\%) (Bucknick et al. 2018).

En cuanto a las mejoras de fuerza en tren inferior los autores concuerdan en el aumento de este parámetro tras la intervención independiente en la diversidad funcional de su población de estudio, esto se puede deber a que todos los autores utilizan equipamiento que favorece la acción de los grupos musculares de extremidad inferior (tapiz rodante, cicloergómetro y elíptica). El estudio de Bartlett et al. (2018) refiere un 11\% mejoría en la prueba levantar y sentarse $(\mathrm{p} \leq 0.05)$ en adultos mayores con artritis reumatoide; mientras que Bellumori et al. (2017) es el autor que logra mayores porcentajes de mejoría en la fuerza extensora de rodilla (34\%) en adultos mayores activos; lo cual se podría explicar debido a que sobre el cicloergómetro se reproduce la acción de dicha musculatura, mientras que los estudios de Buckinx et al. en personas obesas y sedentarias reportan una mejora significativa en la fuerza (isométrica $8 \%$; parase y sentarse:17\%) y potencia muscular de extremidades inferiores (23\%) independientes de la ingesta de proteínas y la suplementación con citrulina adicional (Buckinx et al., 2019b), sin embargo, al igual que para extremidad superior el porcentaje de adultos mayores que responden a la intervención y el porcentaje de ganancia de fuerza es mayor, en aquellos suplementados con citrulina (grupo suplementado con citrulina: $+13,9 \%$ versus grupo placebo: $+3,3 \%$;) (Buckinx et al., 2018). Por último, 2 estudios de Bruseghini et al., difieren de los hallazgos antes mencionados, indicando que el entrenamiento HIIT no mejoró parámetros de fuerza muscular como si lo hizo el entrenamiento isoinercial, que indujo una respuesta hipertrófica de cuádriceps con consiguiente aumento significativo de la fuerza máxima isométrica y dinámica (Bruseghini et al., 2015; Bruseghini et al., 2019).

Del total de artículos revisados, 6 estudios evalúan cambios asociados a variables que caracterizan la movilidad funcional con una prueba en común, que es el Timed up and go (TUG), es así como Bellumori et al. (2017) y Jiménez-García et al. (2019) reportan mejoras significativas y cercanas al 20\% (23 y 18\%, respectivamente) en adultos mayores sanos sin intervención alimentaria, mientras que los estudios de Buckinx et al., indican una mejoría significativa cercana al $10 \%$ la independientes de la ingesta de proteínas y suplementación con citrulina (Buckinx et al., 2019a, Buckinx et al., 2019b, Buckinx et al., 2018) en adultos mayores obesos y/o dinapénicos sedentarios, sin embargo, Bartlett et al. (2017), difiere de los hallazgos anteriores informando una mejora significativa tras aplicar el TUG en adultos mayores con artritis reumatoide, pero sí una mejora significativa de un $8 \%$ en la prueba de velocidad de marcha de 400 metros. Por último, el estudio Bellumori et al. (2017) es el único donde se evalúan los cambios en la función de extremidad superior (test de clavija de 9 orificios). Mientras que son los estudios de Buckinx et al. los que reportan mejoras en variables de desplazamiento de un $13 \%$ en la distancia recorrida en el test de marcha 6 minutos, $15 \%$ en la prueba de alternar pasos y un $87 \%$ en la prueba de balance unipodal, independientes de la ingesta de proteínas y suplementación con citrulina (Buckninx et al., 2019a \& Buckinx et al., 2019b), sin embargo, el mismo autor el 2018 indica que la suplementación con citrulina potencia dichas mejoras (Buckinix et al., 2018).

\section{Efectos del entrenamiento HIIT sobre la calidad de vida}

Solamente 3 autores evaluaron los cambios sobre la calidad de vida tras el programa de intervención HIIT, teniendo en común la aplicación del cuestionario de salud autopercibida SF-36. El estudio de Bellumori et al. (2017) indicó que, si bien existen mejoras en la calidad de vida en pacientes con artritis reumatoidea evaluados con SF-36 posterior al entrenamiento HIIT, estas mejoras no son significativos tras 6 semanas de intervención, complementando estos hallazgos con la aplicación de la escala de confianza $A B C$ que evalúa 
equilibrio y predice futuras caídas para la cual tampoco se produjeron cambios significativos en esta población. El estudio de Benda et al. (2015), reafirma estos resultados, al tampoco encontrar cambios significativos tras aplicar SF-36 más el cuestionario Minnesota en personas mayores con insuficiencia cardiaca tras el doble del tiempo de intervención (12 semanas). Por el contrario, el estudio de Jiménez-García et al. (2019) que intervino a adultos mayores de la comunidad, es el único que reporta beneficios significativos y con un alto tamaño del efecto después del entrenamiento HIIT en comparación con los grupos que entrenaron a moderada intensidad y el grupo control, pero dichos mejorías se produjeron en los dominios del SF-36: salud general, cambios en la salud, vitalidad y funcionamiento físico, además de una mejora significativa en el componente mental de la calidad de vida después de los programas HIIT y a moderada intensidad.

\section{Efectos del entrenamiento HIIT sobre condiciones} clínicas o fisiológicas particulares.

Por último, debido a la diversidad de variable estudiadas en esta revisión, fue posible identificar efectos positivos del entrenamiento HIIT en 4 de 5 estudios para condiciones patológicas o fisiológicas que se indican a continuación:

- Artritis Reumatoidea. Bartlett et al. (2018) demostró que el entrenamiento HIIT reduce la actividad de la enfermedad tras reducir el potencial inflamatorio y el riesgo de sufrir una infección debido a una mayor precisión de la migración de neutrófilos y las funciones bactericidas al mejorar las funciones inmunes innatas.

- Sensibilidad a la Insulina. Søgaard et al. (2019) demostró la existencia de un mayor contenido de ceramidas y ácidos grasos de diacilglicerol en el músculo de sujetos mayores en comparación con los jóvenes, que se podrían relacionar a una mayor sensibilidad a la insulina, condición que tras la intervención con HIIT se redujo mejorando el metabolismo de la glucosa muscular.

- Obesidad dinapénica. Buckinx et al. (2018) demuestran que en adultos mayores con obesidad dinapénica la suplementación con citrulina asociada al entrenamiento HIIT potencia los porcentajes de mejoras con énfasis en la fuerza de tren superior y velocidad de marcha, además de aumentar el porcentaje de pacientes "respondedores" (80\%, versus $50 \%$ de "respondedores" en el grupo placebo).

- Síndrome metabólico. El estudio de Bruseghini et al. (2015) reporta una reducción en los 5 factores de riesgo para desarrollar el síndrome metabólico, los cuales son circunferencia de cintura, presión arterial sistólica, colesterol HDL, niveles de triglicéridos y glucosa tras la intervención HIIT.

- Disfunción mitocondrial. El estudio de Wyckelsma et al. (2017a) revela que entrenamiento HIIT de 12 semanas aumenta el contenido mitocondrial de los adultos mayores y disminuyen las proteínas de la dinámica mitocondrial mitofusina en las fibras tipo II (Mfn2) debido a su mayor eficiencia.

Por último, el estudio de Benda et al. (2015) en adultos mayores con insuficiencia cardiaca es el único que no reporta efectos positivos sobre la condición patológica propia de la enfermedad, como es la estructura y función cardiaca y vascular de estos pacientes.

Entre las limitaciones que se pueden declarar tras esta revisión se encuentran: un solo investigador realizó el análisis de la calidad metodológica de los artículos, sin embargo, la selección y el escrito fue sociabilizado con un grupo de profesionales afines para reducir este sesgo. Los artículos seleccionados para la revisión tuvieron una muestra pequeña y la mayoría no evaluó los efectos a largo plazo (tiempo superior a los 3 meses), esto podría influir en la magnitud de los efectos obtenidos, estimando que un tiempo de intervención y muestra superior podría generar efectos mayores y más claros. Por lo demás, existió un número limitado de estudios para análisis con diferentes características basales de los participantes (sanos y con patologías). Para finalizar, los estudios evaluaron los cambios producidos por el entrenamiento HIIT con diferentes tipos de evaluaciones, lo cual dificulta las comparaciones entre estudios $y$, por ende, tener una mayor claridad de dichos efectos.

\section{Conclusión}

El entrenamiento en HIIT ha demostrado ser una alternativa de intervención viable, segura, con una alta adherencia en adultos mayores activos, sedentarios o que presenten alguna morbilidad. Los efectos positivos de la intervención se alcanzan con entrenamientos a mediano plazo de 6 a 16 semanas, con una frecuencia de 2 a 3 veces a la semana en días no consecutivos que utilizan en su mayoría la modalidad de "HIIT aeróbico" con una duración de la sesión de 30 a 45 minutos. En cuanto a las características de los intervalos, estos fueron a intensidades submáximas de 80 a $95 \%$ y los tiempos de dichos intervalos se distribuyeron desde un mínimo de 30 segundos hasta los 4 minutos, con un total 4 a 10 intervalos por sesión. En cuanto a los efectos del entrenamiento HIIT en personas mayores, 
pueden mencionarse las mejoras sobre la capacidad aeróbica y funcional, además de reducir la carga patológica de la artritis reumatoidea, obesidad y/o dinapenia, con un efecto potencial en la reducción de la sensibilidad a la insulina, características del síndrome metabólico y la disfunción mitocondrial, sin embargo, no existe consenso en la literatura respecto a mejorías en la composición corporal y calidad de vida, es por ello que se requieren una mayor cantidad de estudios experimentales con seguimiento a largo plazo, que permitan dilucidar estas interrogantes.

\section{Agradecimientos}

Al Dr. Pedro Villasana por fomentar la investigación en el departamento de Salud de la Universidad de Los Lagos.

\section{Fuentes de financiación y conflictos de interés}

No hay fuentes de financiación y el autor declara que no posee conflictos de interés para este estudio.

\section{B I B LIO G R A Fí A}

Adamson, S., Lorimer, R., Cobley, J. \& Lloyd, R., Babraj, J. (2014). High intensity training improves health and physical function in middle aged adults. Biology, 3(2), 333-344. doi:10.3390/biology3020333

Bartlett, D. B., Willis, L. H., Slentz, C. A., Hoselton, A., Kelly, L., Huebner, J. L., ... Kraus, W. E. (2018). Ten weeks of high-intensity interval walk training is associated with reduced disease activity and improved innate immune function in older adults with rheumatoid arthritis: a pilot study. Arthritis Research \& Therapy, 20(1), 127. doi:10.1186/ s13075-018-1624-x.

Bellumori, M., Uygur, M., \& Knight, C. A. (2017). High-speed cycling intervention improves rate-dependent mobility in older adults. $\mathrm{Me}$ dicine and Science in Sports and Exercise, 49(1), 106-114. doi:10.1249/ MSS.0000000000001069

Benda, N. M., Seeger, J. P., Stevens, G. G., Hijmans-Kersten, B. T., van Dijk, A. P., Bellersen, L., ...Thijssen, D. H. (2015). Effects of highintensity interval training versus continuous training on physical fitness, cardiovascular function and quality of life in heart failure patients. PLoS One, 10(10), e0141256. doi:10.1371/journal. pone.0141256

Berk, D. R., Hubert, H. B., \& Fries, J. F. (2006). Associations of changes in exercise level with subsequent disability among seniors: a 16-year longitudinal study. The Journals of Gerontology Series A: Biological Sciences and Medical Sciences, 61(1), 97-102. doi:10.1093/gerona/61.1.97

Billat, L. V. (2001). Interval training for performance: a scientific and empirical practice. Sports Medicine, 31(1), 13-31. doi:10.2165/00007256200131010-00002

Bruseghini, P., Calabria, E., Tam, E., Milanese, C., Oliboni, E., Pezzato, A., ... Capelli, C. (2015). Effects of eight weeks of aerobic interval training and of isoinertial resistance training on risk factors of cardiometabolic diseases and exercise capacity in healthy elderly subjects. Oncotarget, 6(19), 16998. doi:10.18632/oncotarget.4031

Bruseghini, P., Capelli, C., Calabria, E., Rossi, A. P., \& Tam, E. (2019). Effects of high intensity interval training and isoinertial training on leg extensors muscle function, structure and intermuscular adipose tissue in older adults. Frontiers in Physiology, 10, 1260. doi:10.3389/ fphys.2019.01260

Buchheit, M. \& Laursen, P.B. (2013). High-intensity interval training, solutions to the programming puzzle: part I: cardiopulmonary emphasis. Sports Medicine, 43(5):313-338. doi:10.1007/s40279-013-0029-x.

Buckinx, F., Gouspillou, G., Carvalho, L., Marcangeli, V., El Hajj Boutros, G., Dulac, M., ... Aubertin-Leheudre, M. (2018). Effect of High-Intensity Interval Training Combined with L-Citrulline Supplementation on Functional Capacities and Muscle Function in Dynapenic-Obese Older Adults. Journal of Clinical Medicine, 7(12), 561. doi:10.3390/ jcm7120561.

Buckinx, F., Gaudreau, P., Marcangeli, V., Boutros, G.E.H., Dulac, M. C., Morais, J. A., \& Aubertin-Leheudre, M. (2019a). Muscle adaptation in response to a high-intensity interval training in obese older adults: effect of daily protein intake distribution. Aging Clinical and Experimental Research, 31(6), 863-874.doi:10.1007/s40520-019-01149-y
Buckinx, F., Marcangeli, V., Pinheiro Carvalho, L., Dulac, M., Hajj Boutros, G., Gouspillou, G., ... Aubertin-Leheudre, M. (2019b). Initial Dietary Protein Intake Influence Muscle Function Adaptations in Older Men and Women Following High-Intensity Interval Training Combined with Citrulline. Nutrients, 11(7), 1685. doi:10.3390/ nu11071685

Campbell, W.W., Kraus, W. E., Powell, K. E., Haskell, W. L., Janz, K. F., Jakicic, J. M., ... Bartlett, D. B. (2019). High-intensity interval training for cardiometabolic disease prevention. Medicine \& Science in Sports \& Exercise, 51(6), 1220-1226. doi:10.1249/MSS.0000000000001934

Carrasco-Poyatos, M., \& Reche-Orene, D. (2018). Efectos de un programa de acondicionamiento físico integrado en el estado funcional de mujeres mayores. Cultura, Ciencia y Deporte, 13(37), 31-38. doi:10.12800/ ccd.v13i37.1036

Chodzko-Zajko, W. J., Proctor, D. N., Singh, M. A. F., Minson, C. T., Nigg, C. R., Salem, G. J., y Skinner, J. S. (2009). Exercise and physical activity for older adults. Medicine \& Science in Sports \& Exercise, 41(7), 1510-1530. doi: 10.1249/MSS.0b013e3181a0c95c

Gibala, M. J., \& McGee, S. L. (2008). Metabolic adaptations to shortterm high-intensity interval training: a little pain for a lot of gain?. Exercise and Sport Sciences Reviews, 36(2), 58-63. doi:10.1097/ JES.0b013e318168ec1f

Gibala, M. J., Little, J. P., MacDonald, M. J., \& Hawley, J. A. (2012). Physiological adaptations to low-volume, high-intensity interval training in health and disease. The Journal of Physiology, 590(5), 1077-1084. doi:10.1113/jphysiol.2011.224725

Hamer, M., Lavoie, K. L., \& Bacon, S. L. (2014). Taking up physical activity in later life and healthy ageing: the English longitudinal study of ageing. British Journal of Sports Medicine, 48(3), 239-43. doi:10.1136/ bjsports-2013-092993

Hautier, C., \& Bonnefoy, M. (2007). Training for older adults. In Annales de Readaptation et de Medicine Physique, 50 (6),475-79. doi:10.1016/j. annrmp.2007.04.018

Helgerud, J., Høydal, K., Wang, E., Karlsen, T., Berg, P., Bjerkaas, M., ... Hoff, J. (2007). Aerobic high-intensity intervals improve V• O2max more than moderate training. Medicine \& Science in Sports \& Exercise, 39(4), 665-671. doi:10.1249/mss.0b013e3180304570

Jiménez-García, J. D., Martínez-Amat, A., De la Torre-Cruz, M. J., Fábrega-Cuadros, R., Cruz-Díaz, D., Aibar-Almazán, A., ... Hita-Contreras, F. (2019). Suspension Training HIIT improves gait speed, strength and quality of life in older adults. International Journal of Sports Medicine, 40(2), 116-124. doi:10.1055/a-0787-1548

Kilpatrick, M. W., Jung, M. E., \& Little, J. P. (2014). High-intensity interval training: a review of physiological and psychological responses. ACSM's Health \& Fitness Journal, 18(5), 11-16. doi:10.1249/ FIT.0000000000000067

Kim, H. K., Suzuki, T., Saito, K., Yoshida, H., Kobayashi, H., Kato, H., \& Katayama, M. (2012). Effects of exercise and amino acid supplementation on body composition and physical function in community-dwelling elderly Japanese sarcopenic women: a randomized con- 
trolled trial. Journal of the American Geriatrics Society, 60(1), 16-23. doi:10.1111/j.1532-5415.2011.03776.x

Landi, F., Marzetti, E., Martone, A. M., Bernabei, R., \& Onder, G (2014). Exercise as a remedy for sarcopenia. Current Opinion in Clinical Nutrition \& Metabolic Care, 17(1), 25-31. doi:10.1097/ MCO.0000000000000018.

Maillard, F., Pereira, B., \& Boisseau, N. (2018). Effect of high-intensity interval training on total, abdominal and visceral fat mass: a meta-analysis. Sports Medicine, 48(2), 269-288. doi:10.1007/s40279-017-0807-y

Manini, T. M., Everhart, J. E., Patel, K. V., Schoeller, D. A., Colbert, L. H., Visser, M., ... Harris, T. B. (2006). Daily activity energy expenditure and mortality among older adults. JAMA, 296(2), 171-79. doi:10.1001/jama.296.2.171

McPhee, J. S., French, D. P., Jackson, D., Nazroo, J., Pendleton, N., \& Degens, H. (2016). Physical activity in older age: perspectives for healthy ageing and frailty. Biogerontology, 17(3), 567-580. doi:10.1007/ s10522-016-9641-0

McRae, G., Payne, A., Zelt, J. G., Scribbans, T. D., Jung, M. E., Little, J. P., \& Gurd, B. J. (2012). Extremely low volume, whole-body aerobicresistance training improves aerobic fitness and muscular endurance in females. Applied Physiology, Nutrition, and Metabolism, 37(6), 11241131. doi:10.1139/h2012-093

Moher, D., Liberati, A., Tetzlaff, J., Altman, D. G., \& Prisma Group. (2010). Preferred reporting items for systematic reviews and metaanalyses: the PRISMA statement. International Journal of Surgery, 8 (5), 336-41. doi:10.1371/journal.pmed.1000097

Brad, R. (2013). High-Intensity Interval Training: Efficient, effective, and a fun way to exercise brought to you by the American College of Sports Medicine. ACSMs Health \& Fitness Journal,7(3):3. doi:10.1249/ FIT.0b013e31828cb21

Sherrington, C., Herbert, R. D., Maher, C. G., \& Moseley, A. M. (2000). PEDro. A database of randomized trials and systematic reviews in physiotherapy. Manual Therapy, 5(4), 223-226. doi:10.1054/ math.2000.0372

Søgaard, D., Baranowski, M., Larsen, S., Taulo Lund, M., Munk Scheuer, C., Vestergaard Abildskov, C., ...Wulff Helge, J. (2019). Muscle-Saturated Bioactive Lipids Are Increased with Aging and Influenced by High-Intensity Interval Training. International Journal of Molecular Sciences, 20(5), 1240. doi:10.3390/ijms20051240

Wisløff,U., Støylen, A., Loennechen, J.P., Bruvold, M., Rognmo, Ø., Haram, P.M., ...Skjaerpe, T (2007). Superior cardiovascular effect of aerobic interval training versus moderate continuous training in heart failure patients: A randomized study. Circulation, 115 (24): 30863094. doi:10.1161/CIRCULATIONAHA.106.675041

Wisløff, U., Ellingsen, Ø., \& Kemi, O. J. (2009). High-intensity interval training to maximize cardiac benefits of exercise training?. Exercise and Sport Sciences Reviews, 37(3), 139-146. doi:10.1097/ JES.0b013e3181aa65fc

Wullems, J. A., Verschueren, S. M., Degens, H., Morse, C. I., \& Onambélé, G. L. (2016). A review of the assessment and prevalence of sedentarism in older adults, its physiology/health impact and nonexercise mobility counter-measures. Biogerontology, 17(3), 547-565. doi:10.1007/s10522-016-9640-1

Wyckelsma, V. L., Levinger, I., McKenna, M. J., Formosa, L. E., Ryan, M. T., Petersen, A. C., ...Murphy, R. M. (2017a). Preservation of skeletal muscle mitochondrial content in older adults: relationship between mitochondria, fibre type and high-intensity exercise training. The Journal of Physiology, 595(11), 3345-59. doi:10.1113/JP273950

Wyckelsma, V. L., Levinger, I., Murphy, R. M., Petersen, A. C., Perry, B. D., Hedges, C. P., ... \& McKenna, M. J. (2017b). Intense interval training in healthy older adults increases skeletal muscle [3H] ouabain-binding site content and elevates $\mathrm{Na}+\mathrm{K}+$-ATPase $\alpha 2$ isoform abundance in Type II fibers. Physiological Reports, 5(7), e13219. doi:10.14814/phy2.13219 